\title{
Epigenetics in the development of diabetic cardiomyopathy
}

\author{
Mark E Pepin ${ }^{1}$ (D) \& Adam R Wende*,1 \\ ${ }^{1}$ Department of Pathology, Division of Molecular \& Cellular Pathology, University of Alabama at Birmingham, Birmingham, AL \\ 35294, USA \\ *Author for correspondence: Tel.: +1 205975 6272; adamwende@uabmc.edu
“ as a convergence of both environmental and genetic influences, epigenetic modifications may program the heart to fail in diabetes."

First draft submitted: 1 January 2019; Accepted for publication: 7 February 2019; Published online: 21 March 2019

Keywords: diabetes $\bullet$ DNA methylation $\bullet$ epigenetics $\bullet$ gene expression $\bullet$ heart failure $\bullet$ metabolism $\bullet$ transcription

\section{Diabetes, heart failure \& underlying changes in gene expression}

Worldwide prevalence of both diabetes mellitus (DM) and heart failure (HF) has exceeded epidemic levels, with current predictions supporting that it will continue to increase [1]. Although DM often coincides with known causes of HF, such as coronary heart disease and hypertension, DM confers a roughly fourfold increased risk of HF independent of its clinical covariates [2]. This increased risk also occurs irrespective of DM subtype, implicating the shared features of both Type 1 and Type 2 DM. Among these, perturbation to the systemic metabolic milieu are of particular concern.

Diabetic cardiomyopathy (DCM) describes the clinical syndrome of HF wherein DM is the only discernable cause of impaired cardiac contractility or compliance [3]. Despite its controversial origins as a distinct pathology [4], growing evidence from both human and rodent studies supports an inverse association between glycemic control and risk of cardiovascular complications. Furthermore, owing to the marked disruptions to cardiac metabolic substrate utilization in HF, some propose that - even in the absence of DM - HF is a metabolic disease of the heart. In response to pressure overload or ischemic injury, the failing heart exhibits an abnormal metabolic preference for carbohydrates while inhibiting fatty acid metabolism [5]. This physiologic shift toward carbohydrate utilization is accompanied by the reactivation of numerous developmental gene programs wherein glucose is the preferred fuel [6]. In the context of diabetes, however, the failing heart has been shown to differ from the 'fetallike' metabolic program of HF. For over half a century, DCM has been known to disrupt cardiac metabolism by decreasing myocardial glucose consumption, modestly increasing ketone metabolism, and significantly increasing its utilization of fatty acids [7]. These observations have posed the challenge of identifying the underlying mechanisms that control cardiac metabolism and, in particular, those which are susceptible to disruption by diabetes. The rapidly developing field of epigenetics has uncovered new insights into the mechanism of metabolic gene regulation, offering exciting therapeutic potential to reverse these pathologic 'fetal' gene programs and restore cardiac function. This commentary will briefly address the current evidence of epigenetic regulation in HF, as well as continuing efforts to define therapeutic targets.

\section{Emerging mechanisms of cardiac gene regulation: epigenetics}

Owing to the diversity of environmental, lifestyle and genetic factors as modifiers of heart failure susceptibility, identifying the molecular underpinnings of HF pathogenesis has proven very difficult. While precision-based genomic studies have provided insight into heritable forms of HF, epigenetics addresses the confounding effects of environmental exposures on genetic susceptibility for complex diseases, as highlighted by the NIH Roadmap Epigenomics Program [8]. As a field, epigenetics is the study of stable - yet reversible - mechanisms of gene regulation. In disease, these epigenetic modifications contribute to a long-term disruption of gene expression, yet retain potential plasticity through dietary, exercise and evolving pharmaceutical intervention. With that said,

Future $\because$ Medicine 
how might we use the knowledge of metabolic phenotypes defining the different HF etiologies? One way is via the signaling of the metabolites themselves to the regulation of neighboring pathways, as nicely reviewed by Gut and Verdin [9]. Within this review, the authors summarize current evidence to conclude that 'understanding the influence of nutrients, metabolites and other environmental factors on the metabolic landscape and its influence on the epigenome will probably open up new therapeutic strategies'. Earlier work has already provided the initial steps to define these epigenetic mechanisms, specifically differential DNA and histone methylation, occurs in human end-stage cardiomyopathy [10].

In addition to the role of individual metabolites themselves, it is also theorized that other ancillary metabolic components, such as coupling of oxygen consumption, contribute to the regulation of cardiac metabolism in DCM. Specifically, it has been suggested that HF uncouples cardiac glycolysis from subsequent glucose oxidation, leading to 'cardiac inefficiency' as it relates to oxygen consumed for a given workload [11]. Although a slightly different connection, we have recently found in human heart failure cardiac samples a potential link between regulation of oxygen sensing via HIF-1 $\alpha$ and regulation of epigenetic pathways to change gene expression in a manner consistent with this switch from oxidative to glycolytic metabolism [12]. In this study, we found that the polycomb methyltransferase EZH2 gene was upregulated consistent with HIF-1 $\alpha$-mediated activation and when associated with the transcription factor FOXM1 protein revealed induction of glycolytic genes, versus a linkage with suppression of oxidative metabolic genes when associated with PRC2 protein complex. Along these lines, we also found increased promoter methylation of the transcription factor KLF15 gene, which appeared to be directly regulated by EZH2 protein induction in vitro following overexpression studies in cardiac cells. Although these studies reveal a strong correlation between DNA methylation and gene expression, they are applicable to heart failure independent of diabetes, and the known differences in metabolic utilization strongly support that ischemic heart failure is distinct from DCM.

\section{Epigenetics \& diabetic cardiomyopathy}

The relationship between metabolic disruption and disease susceptibility is most apparent in diabetic complications. In fact, it has been suggested for decades that the relationship between glycemic control and development of organ dysfunction may be directly related and have therefore been termed 'metabolic memory' or, more specifically, 'glycemic memory' [13]. Evidence of glycemic memory was first developed following completion of the Diabetes Control and Complications Trial (DCCT) of Type 1 diabetic patients, in which patients were randomized into two cohorts of 'conventional control' or 'intense control' glycemic maintenance groups. The study was halted prematurely due to compelling evidence that tight glycemic control ( $\sim 2 \%$ lower HbA1c) resulted in significantly lower diabetic complications, including cardiovascular disease. The investigative team designed a follow-up study, called the Epidemiology of Diabetes Interventions and Complications (EDIC), which examined the longevity of disproportionately elevated risk in patients randomized to the formerly conventional control group. Despite equivalent glycemic control throughout the follow-up study, there is evidence for worse outcomes related to development of both microvascular and macrovascular complications that persist for decades in the initial conventional treatment group [14,15]. Similar findings in Type 2 diabetic patients were made in both the United Kingdom Prospective Diabetes Study (UKPDS) and the Veteran's Administration Diabetes Trial (VADT), which discovered improved patient outcomes (fewer complications) in response to intensive glycemic control $[16,17]$. These trials concluded that optimal glycemic control involves balancing patient risk of diabetic complications; however, it has also been noted that caution needs to be taken to reduce severe hypoglycemic episodes [18]. Regardless, it is now clear that glycemic instability contributes to long-term diabetic complications. Although the epigenetic underpinnings of these clinical observations remain incompletely understood, these mechanisms may serve as the missing lens needed to bring individual contributors of DCM risk into focus. Furthermore, as a convergence of both environmental and genetic influences, epigenetic modifications may program the heart to fail in diabetes.

Although many remaining questions exist regarding the connection between diabetes, epigenetics and CVD, several pioneering studies offer preliminary insight. One of the first direct examples of glucose-mediated changes in epigenetics and gene expression came from El-Osta et al. [19], where aortic endothelial cells transiently treated with high glucose were found to induce stable histone mono-methylation associated with gene expression consistent with a proatherogenic state. Furthermore, studies of patients from the DCCT/EDIC trial have reported a relationship between DNA methylation and inverse gene expression in targets related to diabetic complications, such as the TXNIP gene [20]. In addition to these examples of histone and DNA methylation dynamics in diabetic complications, the role of noncoding RNAs is an active area of investigation. For example, studies in streptozotocin- 
induced diabetic mice revealed that even after blood glucose was normalized, changes in the miRNA landscape in the heart remained the same [21]. Together these select examples support the persistent nature of cardiovascular risk even after maintaining euglycemia.

\section{Conclusion \& future perspective}

The notion that epigenetics encodes a glycemic memory instills an almost fatalistic outlook on diabetic outcomes, perhaps to the point of questioning why continued treatment is even warranted. After all, why should anyone amend their risk profile after the underlying molecular features of disease have already been established? This question logically surfaces in the context of any chronic disease where manifestations of disease pathology are deemed 'irreversible' (e.g., cigarette smoking and ischemic heart disease). To this, we offer two closing thoughts. First, even if medical and/or lifestyle modifications cannot easily reverse the epigenetic predisposition for disease complications, adjusting the risk profile significantly reduces the continued accrual of the underlying disease pathology. Furthermore, just as the diabetic milieu can epigenetically disrupt cardiac gene expression, lifestyle and medical interventions may too reverse these pathological programs, albeit insufficient to re-establish a 'healthy' state. Future studies should determine the extent to which exercise and diet modify the cardiac epigenome in the setting of DCM. Second, although current treatment options do not exist to address the epigenetic mechanisms of DCM, development of epigenetic therapies may 'reset' this metabolic memory. As mentioned previously, we recently identified the epigenetic regulator $\mathrm{EZH} 2$ protein as a likely metabolic regulator in our study of ischemic HF. Interestingly, multiple clinical trials are underway to determine the utility of EZH2 activity inhibition in cancer therapy, which has also recently been demonstrated as an effective therapy in rodent models to hepatic fibrosis [22]. Therefore, the re-purposing of these US FDA approved small molecule inhibitors in ischemic HF to reprogram cardiac metabolism would support a similar treatment paradigm of epigenetic interventions for diabetic complications, including diabetic cardiomyopathy.

In summary, growing evidence supports that diabetes drives an epigenetic reprogramming of cardiac metabolism. Once identified, the epigenetic regulators of cardiac metabolism will resolve a longstanding controversy regarding the exact nature - and even existence - of metabolic memory. Furthermore, these studies would define the molecular targets of future endeavors to reset the epigenetic susceptibility for, perhaps even the manifestation of, diabetic cardiomyopathy.

\section{Acknowledgements}

Owing to the brief nature of this commentary and reference limits, the authors wish to apologize to other authors whose work could not be cited but none-the-less provide significant influence to the shaping of the views presented herein. As such we have cited a number of reviews and refer the reader to the valuable articles contained within each.

\section{Financial \& competing interests disclosure}

Work of the authors was supported by NIH grants: R01 HL133011 (to AR Wende) and F30 HL137240 (to ME Pepin). The authors have no other relevant affiliations or financial involvement with any organization or entity with a financial interest in or financial conflict with the subject matter or materials discussed in the manuscript apart from those disclosed.

No writing assistance was utilized in the production of this manuscript.

\section{References}

1. Cho NH, Shaw JE, Karuranga S et al. IDF diabetes atlas: global estimates of diabetes prevalence for 2017 and projections for 2045 . Diabetes Res. Clin. Pract. 138, 271-281 (2018).

2. Kannel WB, Hjortland M, Castelli WP. Role of diabetes in congestive heart failure: the Framingham study. Am. J. Cardiol. 34(1), 29-34 (1974).

3. Bugger H, Abel ED. Molecular mechanisms of diabetic cardiomyopathy. Diabetologia 57(4), 660-671 (2014).

4. Hölscher M, Bode C, Bugger H. Diabetic cardiomyopathy: does the type of diabetes matter? Int. J. Mol. Sci. 17(12), 2136-2145 (2016).

5. Sansbury BE, Demartino AM, Xie Z et al. Metabolomic analysis of pressure-overloaded and infarcted mouse hearts. Circ. Heart Fail. 7(4), 634-642 (2014).

6. Taegtmeyer H, Sen S, Vela D. Return to the fetal gene program: a suggested metabolic link to gene expression in the heart. Ann. N. Y. Acad. Sci. 1188, 191-198 (2010).

7. Ungar I, Gilbert M, Siegel A, Blain JM, Bing RJ. Studies on myocardial metabolism: IV. Myocardial metabolism in diabetes. Am. J. Med. 18(3), 385-396 (1955). 
8. Romanoski CE, Glass CK, Stunnenberg HG, Wilson L, Almouzni G. Epigenomics: roadmap for regulation. Nature 518(7539), 314-316 (2015).

9. Gut P, Verdin E. The nexus of chromatin regulation and intermediary metabolism. Nature 502(7472), 489-498 (2013).

10. Movassagh M, Choy M-K, Knowles DA et al. Distinct epigenomic features in end-stage failing human hearts. Circulation 124(22), 2411-2422 (2011).

11. Lopaschuk GD. Metabolic modulators in heart disease: past, present, and future. Can. J. Cardiol. 33(7), 838-849 (2017).

12. Pepin ME, Ha C-M, Crossman DK et al. Genome-wide DNA methylation encodes cardiac transcriptional reprogramming in human ischemic heart failure. Lab. Invest. 99, 371-386 (2019).

13. Cooper ME, El-Osta A. Epigenetics: mechanisms and implications for diabetic complications. Circ. Res. 107(12), 1403-1413 (2010).

14. EDIC Research Group. Epidemiology of diabetes interventions and complications (EDIC). Design, implementation, and preliminary results of a long-term follow-up of the diabetes control and complications trial cohort. Diabetes Care 22(1), 99-111 (1999).

15. Diabetes Control and Complications Trial (DCCT)/, Epidemiology of Diabetes Interventions and Complications (EDIC) Study Research Group. Intensive diabetes treatment and cardiovascular outcomes in Type 1 diabetes: The DCCT/EDIC study 30-year follow-up. Diabetes Care 39(5), 686-693 (2016).

16. Holman RR, Paul SK, Bethel MA, Matthews DR, Neil HA. 10-year follow-up of intensive glucose control in Type 2 diabetes. N. Engl. J. Med. 359(15), 1577-1589 (2008).

17. Agrawal L, Azad N, Bahn GD et al. Long-term follow-up of intensive glycaemic control on renal outcomes in the Veterans Affairs Diabetes Trial (VADT). Diabetologia 61(2), 295-299 (2018).

18. Zhou JJ, Schwenke DC, Bahn G, Reaven P. Glycemic variation and cardiovascular risk in the veterans affairs diabetes trial. Diabetes Care 41(10), 2187-2194 (2018).

19. El-Osta A, Brasacchio D, Yao D et al. Transient high glucose causes persistent epigenetic changes and altered gene expression during subsequent normoglycemia. J. Exp. Med. 205(10), 2409-2417 (2008).

20. Chen Z, Miao F, Paterson AD et al. Epigenomic profiling reveals an association between persistence of DNA methylation and metabolic memory in the DCCT/EDIC Type 1 diabetes cohort. Proc. Natl Acad. Sci. USA 113(21), E3002-E3011 (2016).

21. Costantino S, Paneni F, Lüscher TF, Cosentino F. MicroRNA profiling unveils hyperglycaemic memory in the diabetic heart. Eur. Heart J. 37(6), 572-576 (2016).

22. Martin-Mateos R, De Assuncao TM, Arab JP et al. Enhancer of zeste homologue 2 inhibition attenuates TGF- $\beta$. Cellular Mol. Gastroenterol. Hepatol. 7(1), 197-209 (2019). 